\title{
Catalysis by Ionic Liquid. A Green Protocol for the Stereoselective Debromination of vicinal-Dibromides by $[\mathrm{pmIm}] \mathrm{BF}_{4}$ under Microwave Irradiation
}

\author{
Brindaban C. Ranu* and Ranjan Jana
}

Supporting Information

Table of Contents

Spectroscopic (IR, ${ }^{1} \mathrm{H}$ NMR and ${ }^{13} \mathrm{C}$ NMR) Data of Products Page no.

in Entries 14-25, Table $1 \quad$ S2-S4

${ }^{13} \mathrm{C}$ NMR Spectra of all compounds $\quad$ S5-S16 
Spectroscopic (IR, ${ }^{1} \mathrm{H}$ NMR and ${ }^{13} \mathrm{C}$ NMR) Data of Products in Entries 14-25, Table 1

entry 14. Pale yellow solid, m.p. $61{ }^{\circ} \mathrm{C}$; $\operatorname{IR}(\mathrm{KBr}) 1656,1600,1577,1512,1263 \mathrm{~cm}^{-}$ ${ }^{1}$; ${ }^{1} \mathrm{H}$ NMR $\left(300 \mathrm{MHz}, \mathrm{CDCl}_{3}\right) \delta 3.81$ (s, 3H), 6.90-6.94 (m, 2H), 7.41 (d, $J=15.7 \mathrm{~Hz}, 1 \mathrm{H}), 7.45-7.59(\mathrm{~m}, 5 \mathrm{H}), 7.79(\mathrm{~d}, J=15.7 \mathrm{~Hz}, 1 \mathrm{H}), 8.00-8.03$ $(\mathrm{m}, 2 \mathrm{H}) ;{ }^{13} \mathrm{C}$ NMR (75 MHz) $\delta 55.2,114.2(2 \mathrm{C}), 119.5,127.3,128.2(2 \mathrm{C})$, 128.4 (2C), 130.1 (2C), 131.8, 138.3, 144.5, 161.5, 190.3.

entry 15 Pale yellow solid, m.p. $108{ }^{\circ} \mathrm{C}$; $\operatorname{IR}(\mathrm{KBr}) 1658,1606,1589,1573,1018$, $777 \mathrm{~cm}^{-1} ;{ }^{1} \mathrm{H}$ NMR $\left(300 \mathrm{MHz}, \mathrm{CDCl}_{3}\right) \delta 5.99(\mathrm{~s}, 2 \mathrm{H}), 6.81(\mathrm{~d}, J=8.0 \mathrm{~Hz}$, 1H), 7.07-7.14 (m, 2H), 7.34 (d, $J=15.63 \mathrm{~Hz}, 1 \mathrm{H}), 7.44-7.55(\mathrm{~m}, 3 \mathrm{H})$, $7.71(\mathrm{~d}, J=15.63 \mathrm{~Hz}, 1 \mathrm{H}), 7.96-7.99(\mathrm{~m}, 2 \mathrm{H}) ;{ }^{13} \mathrm{C} \mathrm{NMR}(75 \mathrm{MHz}) \delta$ 101.6, 106.6, 108.6, 120.0, 125.2, 128.3 (2C), 128.5 (2C), 129.3, 132.6, $138.3,144.6,148.3,149.8,190.3$.

entry 16 Viscous brown liquid, IR(neat) 1658, 1600, $1222 \mathrm{~cm}^{-1} ;{ }^{1} \mathrm{H}$ NMR (300 $\left.\mathrm{MHz}, \mathrm{CDCl}_{3}\right) \delta 6.43-6.45(\mathrm{~m}, 1 \mathrm{H}), 6.67(\mathrm{~d}, J=2.01 \mathrm{~Hz}, 1 \mathrm{H}), 7.40-7.53$ $(\mathrm{m}, 5 \mathrm{H}), 7.57(\mathrm{~d}, J=15.4 \mathrm{~Hz}, 1 \mathrm{H}), 7.99(\mathrm{~d}, J=8.1 \mathrm{~Hz}, 2 \mathrm{H}) ;{ }^{13} \mathrm{C}$ NMR $(75$ MHz) $\delta 112.7,116.3,119.2,128.4$ (2C), 128.6 (2C), 130.7, 132.8, 138.1, 145.0, 151.6, 189.7.

entry 17 Brown solid, m.p. $42-44{ }^{\circ} \mathrm{C}$; $\mathrm{IR}(\mathrm{KBr}) 1652,1583,1573,1215 \mathrm{~cm}^{-1} ;{ }^{1} \mathrm{H}$ NMR (300 MHz, $\left.\mathrm{CDCl}_{3}\right) \delta 6.89(\mathrm{t}, J=4.9 \mathrm{~Hz}, 1 \mathrm{H}), 7.12-7.28(\mathrm{~m}, 3 \mathrm{H})$, 7.30-7.41 (m, 3H), $7.76(\mathrm{~d}, J=15.3 \mathrm{~Hz}, 1 \mathrm{H}), 7.82(\mathrm{~d}, J=7.32 \mathrm{~Hz}, 2 \mathrm{H})$; ${ }^{13} \mathrm{C}$ NMR (75 MHz) $\delta 120.7,128.1$ (3C), 128.6 (2C), 128.8, 132.0, 132.7, 137.1, 138.0, 140.3, 189.8 .

entry 18 Pale yellow solid, m.p. $82{ }^{\circ} \mathrm{C}$; $\operatorname{IR}(\mathrm{KBr}) 1662,1602,1575,1446,1348$, 1006, $781 \mathrm{~cm}^{-1} ;{ }^{1} \mathrm{H}$ NMR $\left(300 \mathrm{MHz}, \mathrm{CDCl}_{3}\right) \delta$ 7.47-7.57 (m, 6H), $7.63(\mathrm{~d}$, $J=15.3 \mathrm{~Hz}, 1 \mathrm{H}), 7.85-7.91(\mathrm{~m}, 3 \mathrm{H}), 8.06(\mathrm{~d}, J=8.7 \mathrm{~Hz}, 2 \mathrm{H}), 8.23(\mathrm{~d}, J=$ $8.1 \mathrm{~Hz}, 1 \mathrm{H}), 8.66(\mathrm{~d}, J=15.3 \mathrm{~Hz}, 1 \mathrm{H}) ;{ }^{13} \mathrm{C}$ NMR $(75 \mathrm{MHz}) \delta 123.3$, $124.5,124.9,125.2,126.1,126.8,128.4$ (2C), 128.5 (2C), 128.6, 130.6, 131.6, 132.2, 132.7, 133.5, 138.0, 141.6, 190.1 . 
entry 19 Pale yellow liquid; IR(neat) 1674, 1600, 1575, 1508, $1255 \mathrm{~cm}^{-1} ;{ }^{1} \mathrm{H}$ NMR $\left(300 \mathrm{MHz}, \mathrm{CDCl}_{3}\right) \delta$ 4.37-4.41 (m, 2H), 5.09-5.26 (m, 2H), 5.79-5.88 (m, $1 \mathrm{H})$, 6.71-6.80 (m, 2H), 7.10-7.26 (m, 2H), $7.35(\mathrm{~d}, J=15.6 \mathrm{~Hz}, 1 \mathrm{H})$, 7.42-7.45 (m, 1H), $7.60(\mathrm{~d}, J=15.6 \mathrm{~Hz}, 1 \mathrm{H}), 7.71(\mathrm{~d}, J=8.43 \mathrm{~Hz}, 2 \mathrm{H})$, $7.83(\mathrm{~d}, J=8.76 \mathrm{~Hz}, 2 \mathrm{H}) ;{ }^{13} \mathrm{C}$ NMR $(75 \mathrm{MHz}) \delta 68.9,114.5(2 \mathrm{C}), 118.8$, $121.8,127.5,128.3,128.4,128.9,130.3$ (2C), 130.7, 132.3, 132.5, 137.5, $143.9,162.8,188.6$.

entry 20 Light red solid, m.p. $60{ }^{\circ} \mathrm{C}$; $\mathrm{IR}(\mathrm{KBr}) 1483,755 \mathrm{~cm}^{-1} ;{ }^{1} \mathrm{H}$ NMR $(300 \mathrm{MHz}$, $\left.\mathrm{CDCl}_{3}\right) \delta$ 7.33-7.37 (m, 6H), 7.53-7.57 (m, 4H); ${ }^{13} \mathrm{C} \mathrm{NMR}(75 \mathrm{MHz}) \delta$ 89.4 (2C), 123.3 (2C), 128.3 (2C), 128.4 (4C), 131.6 (4C).

entry 21 Colorless solid, m.p. $112{ }^{\circ} \mathrm{C}$; $\mathrm{IR}(\mathrm{KBr}) 1485,756 \mathrm{~cm}^{-1} ;{ }^{1} \mathrm{H}$ NMR $(300$ $\left.\mathrm{MHz}, \mathrm{CDCl}_{3}\right) \delta$ 7.34-7.36 (m, 3H), 7.46-7.49 (m, 2H), 7.56-7.59 (m, 3H), 7.78-7.81 (m, 3H), $8.05(\mathrm{~s}, 1 \mathrm{H}) ;{ }^{13} \mathrm{C}$ NMR $(75 \mathrm{MHz}) \delta 87.7,94.5,121.0$, $123.5,125.4,126.3,126.6,126.9,128.5$ (2C), 128.6, 128.7, 128.9, 130.5, $131.8(2 \mathrm{C}), 133.3,133.4$.

entry 22 Pale yellow solid, m.p. $114{ }^{\circ} \mathrm{C}$; $\mathrm{IR}(\mathrm{KBr}) 1647,1591,1575,1448,1315$, $696 \mathrm{~cm}^{-1} ;{ }^{1} \mathrm{H}$ NMR $\left(300 \mathrm{MHz}, \mathrm{CDCl}_{3}\right) \delta$ 7.49-7.54 (m, 4H), 7.66-7.69 (m, $2 \mathrm{H}), 8.14-8.17(\mathrm{~m}, 4 \mathrm{H}) ;{ }^{13} \mathrm{C}$ NMR $(75 \mathrm{MHz}) \delta 85.7$ (2C), 128.9 (4C), 129.7 (4C), 135.1 (2C), 135.7 (2C), 176.4 (2C).

entry 23 Pale yellow solid, m.p. $123{ }^{\circ} \mathrm{C}$; $\mathrm{IR}(\mathrm{KBr}) 1653,1641,1622,1596,1282$, $696 \mathrm{~cm}^{-1} ;{ }^{1} \mathrm{H}$ NMR $\left(300 \mathrm{MHz}, \mathrm{CDCl}_{3}\right) \delta$ 7.43-7.59 (m, 5H), $7.81(\mathrm{t}, J=$ $8.3 \mathrm{~Hz}, 2 \mathrm{H}), 7.91(\mathrm{~d}, J=7.7 \mathrm{~Hz}, 1 \mathrm{H}), 8.04(\mathrm{~d}, J=8.3 \mathrm{~Hz}, 1 \mathrm{H}), 8.13$ (d, $J=$ $7.4 \mathrm{~Hz}, 2 \mathrm{H}), 8.64(\mathrm{~s}, 1 \mathrm{H}) ;{ }^{13} \mathrm{C}$ NMR $(75 \mathrm{MHz}) \delta 85.6,85.9,123.1,127.1$, $127.8,128.8$ (3C), 129.5, 129.6 (2C), 129.8, 132.1, 133.2, 133.5, 135.0, 135.7, 136.3, 176.2, 176.5 .

entry 24 Pale yellow solid, m.p. $134{ }^{\circ} \mathrm{C}$; $\mathrm{IR}(\mathrm{KBr}) 1643,1629,1595,1577,1494$, $1448,1271,696 \mathrm{~cm}^{-1} ;{ }^{1} \mathrm{H}$ NMR $\left(300 \mathrm{MHz}, \mathrm{CDCl}_{3}\right) \delta 6.86(\mathrm{~d}, J=16.3 \mathrm{~Hz}$, $1 \mathrm{H})$, 7.34-7.39 (m, 3H), 7.45-7.55 (m, 4H), 7.59-7.64 (m, 1H), 7.83-7.88 $(\mathrm{d}, J=16.3 \mathrm{~Hz}, 1 \mathrm{H}), 8.10-8.13(\mathrm{~d}, J=8.0 \mathrm{~Hz}, 2 \mathrm{H}) ;{ }^{13} \mathrm{C} \mathrm{NMR}(75 \mathrm{MHz}) \delta$ 
84.4, 85.5, 127.5, 128.9 (3C), 129.0, 129.1 (2C), 129.7 (2C), 131.8, 133.4, 135.0, 135.7, 150.6, 176.4, 176.6.

entry 25 Colorless solid, m.p. $115^{\circ} \mathrm{C}$; IR(KBr) 1479, 1294, 1230, 1004, $800 \mathrm{~cm}^{-1}$; ${ }^{1} \mathrm{H}$ NMR $\left(300 \mathrm{MHz}, \mathrm{CDCl}_{3}\right) \delta 4.77(\mathrm{~s}, 4 \mathrm{H}), 6.86(\mathrm{~d}, J=8.8 \mathrm{~Hz}, 2 \mathrm{H}), 7.08-$ $7.12\left(\mathrm{dd}, J_{1}=8.8 \mathrm{~Hz}, J_{2}=2.5 \mathrm{~Hz}, 4 \mathrm{H}\right), 7.37(\mathrm{~d}, J=2.5 \mathrm{~Hz}, 2 \mathrm{H}) ;{ }^{13} \mathrm{C} \mathrm{NMR}$ $(75 \mathrm{MHz}) \delta 56.9$ (2C), 82.2 (2C), 115.0 (2C), 126.7 (2C), $127.2(2 \mathrm{C})$, $130.0(4 \mathrm{C}), 151.5(2 \mathrm{C})$. 
RJ-188I-13C (SR)

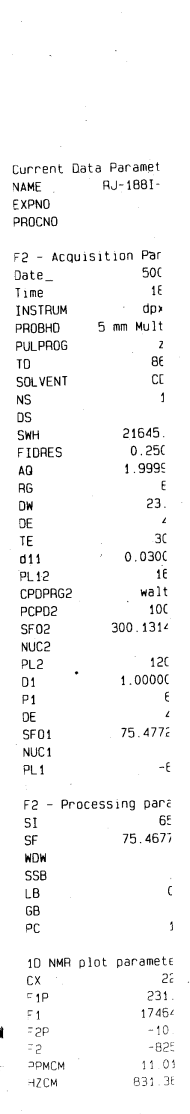

RJ-181I-13C (SKD)

$\begin{array}{ll}\text { F2 - Acquisition Par } \\ \text { Date_ } & 50 \mathrm{C}\end{array}$

Date-
Time
INSTRUM
PROBHD
PPLPOOG

$\begin{array}{lr}\text { PULPPOG } & \text { 2 } \\ \text { ID } & \text { IE } \\ \text { SO } & \text { CI }\end{array}$

言

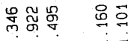

V

SOLVE
NS
DS
SWH

\begin{tabular}{lr} 
DS & \\
SWH & 21645 \\
F IDRES & 0.256 \\
AO & 1.9996 \\
\hline
\end{tabular}

.
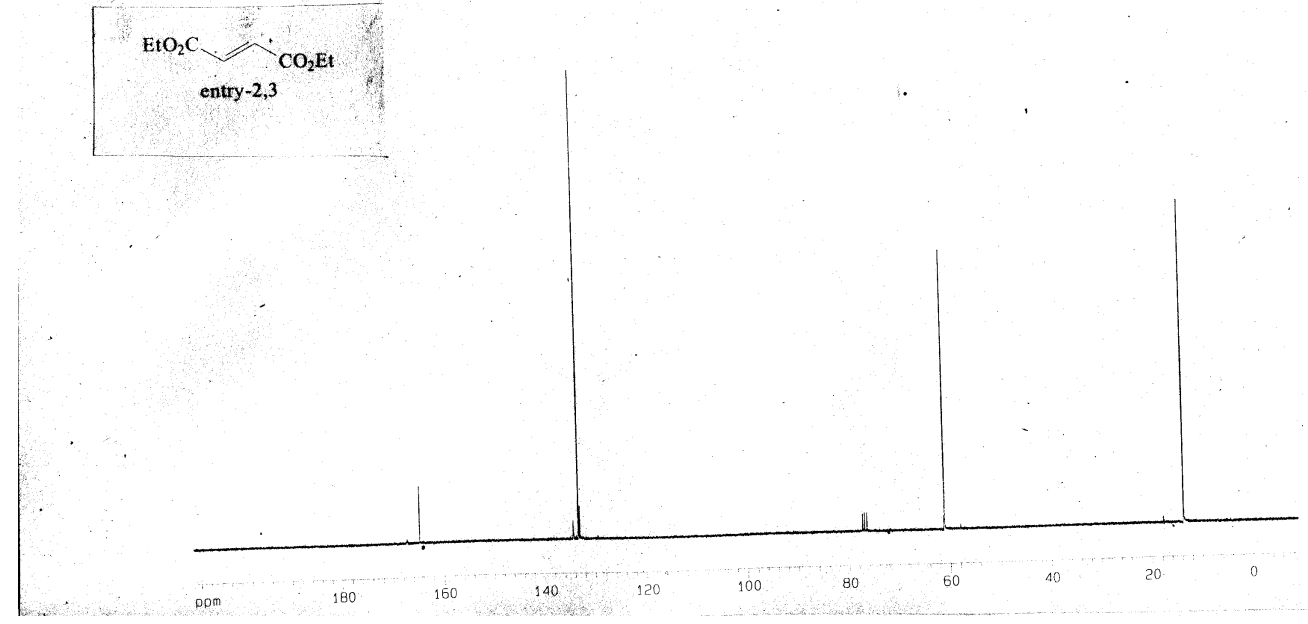


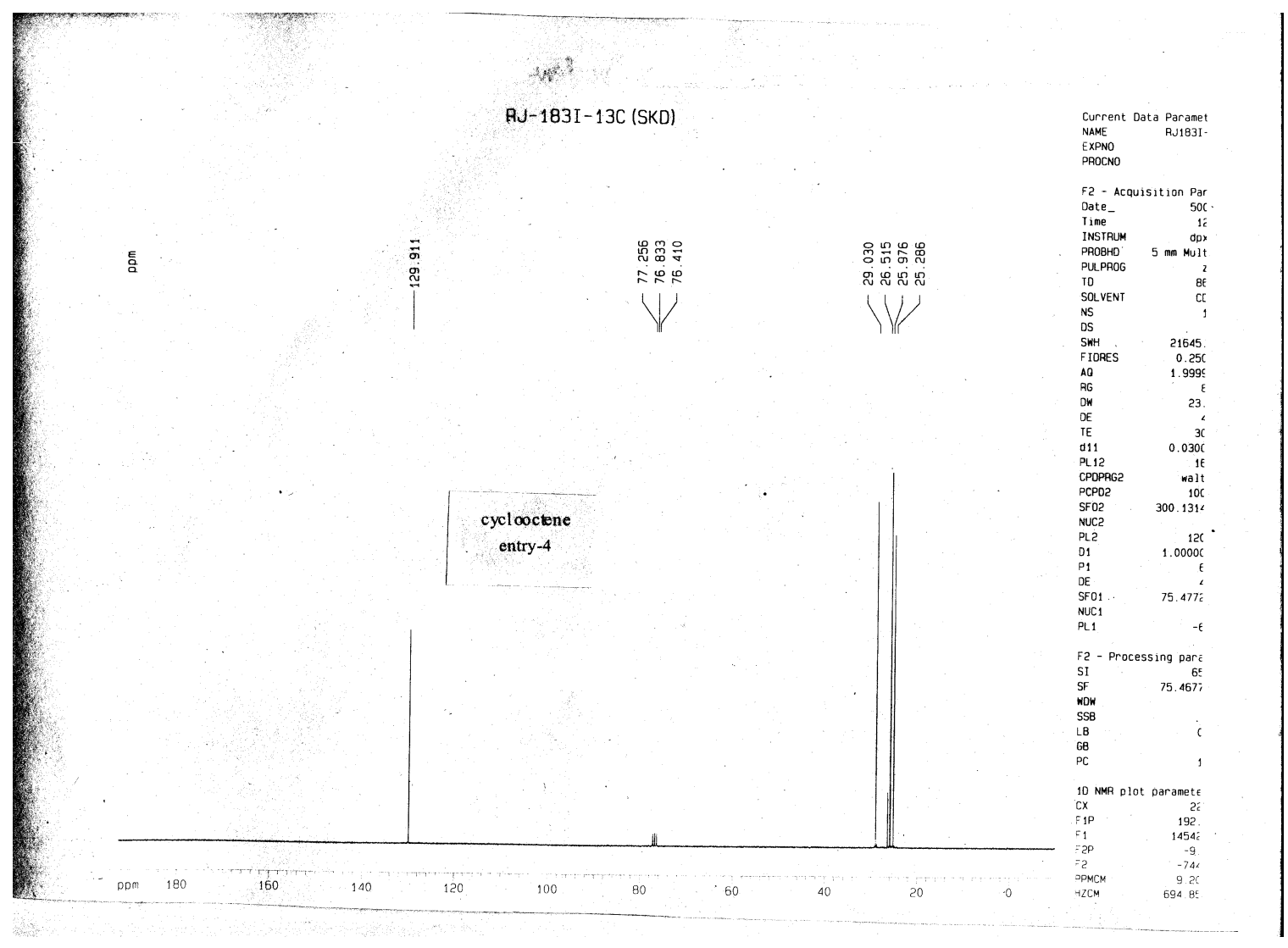




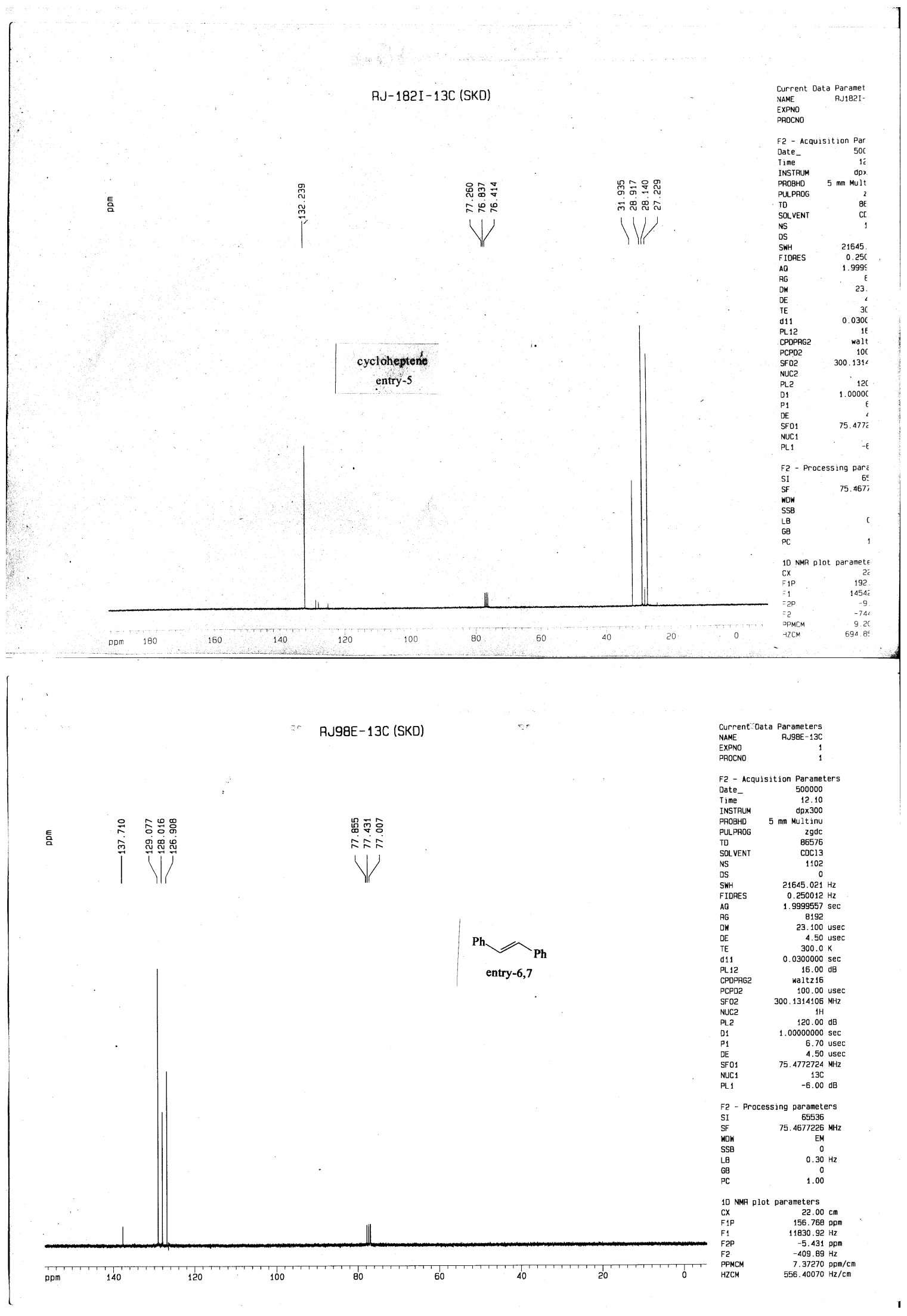




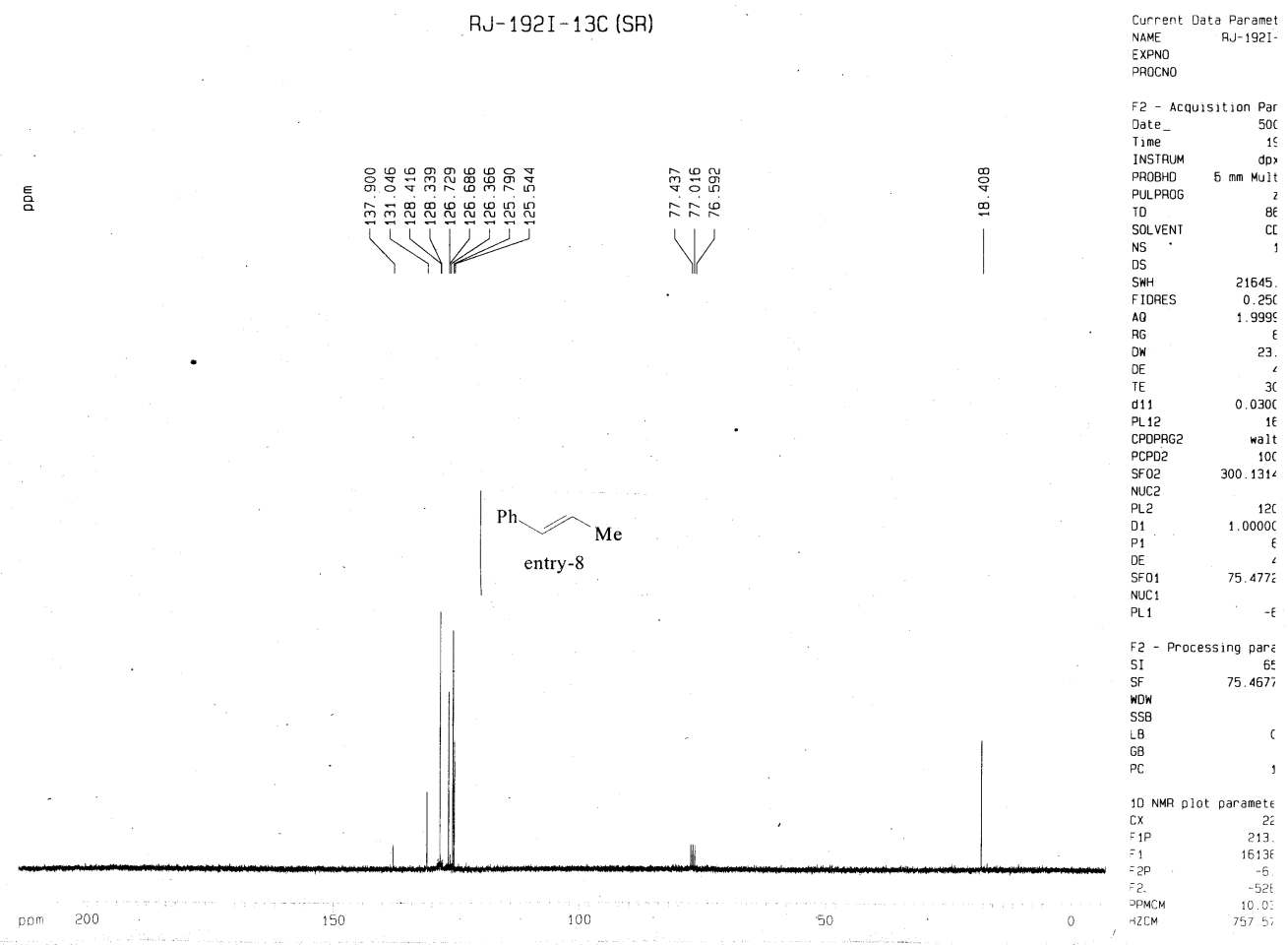

$R J-180 I-13 C$ (SKD)

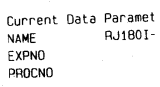

$\mathrm{F} 2-$ Acquisition $\mathrm{Par}$
$50 \mathrm{C}$

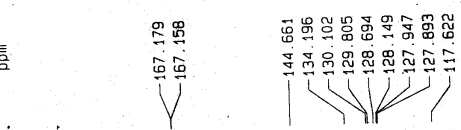

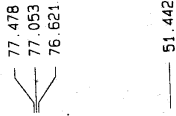

Time

$\begin{array}{lr}\text { PULPROG } & 2 \\ \text { TO } & 8 E \\ \text { SO } & \text { CE }\end{array}$

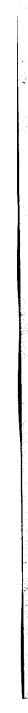

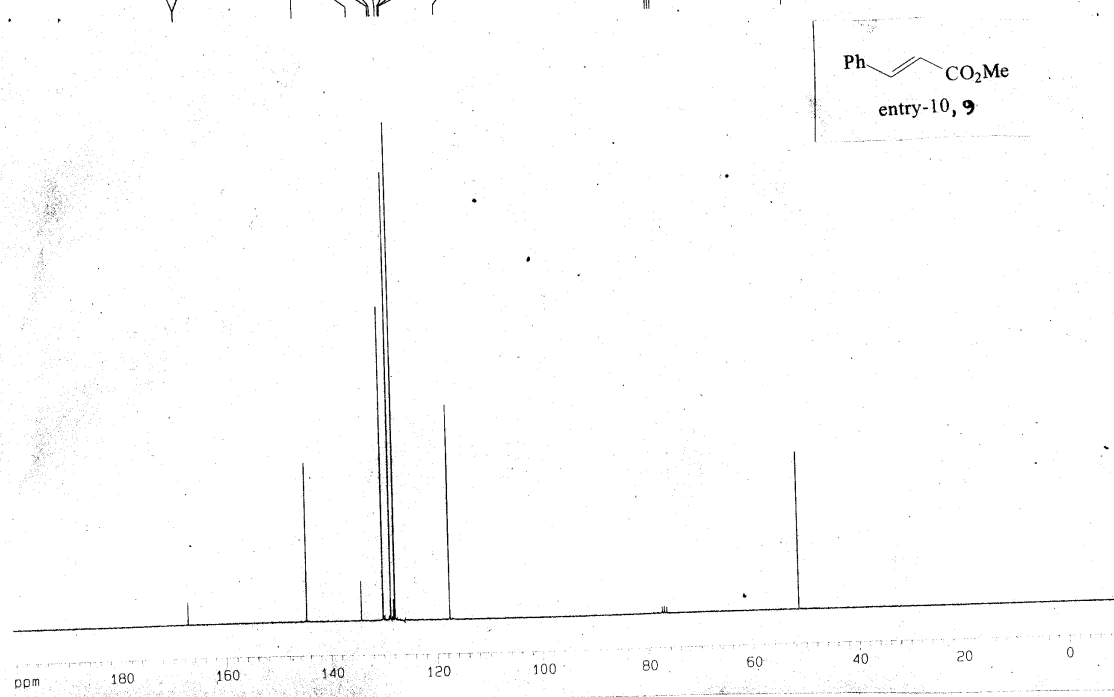

NS
DS
SWH

21645 .

\begin{tabular}{lr} 
SWH & 21645. \\
FIDAES & $0.25 \mathrm{C}$ \\
AQ & 1.9995 \\
\hline &
\end{tabular}

AO
AO
Do

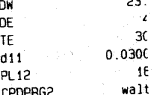

CPDPAG
PCPO2

SFO2
NUC2

1126
012
01

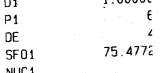

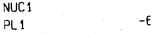

F2 - Processing pare
SI
SF
SF

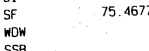

$S S B$
$\angle B$
$6 B$

10 NMA plot paramete

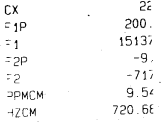

180

. 
RJ $-186 I-13 C$ (SR)

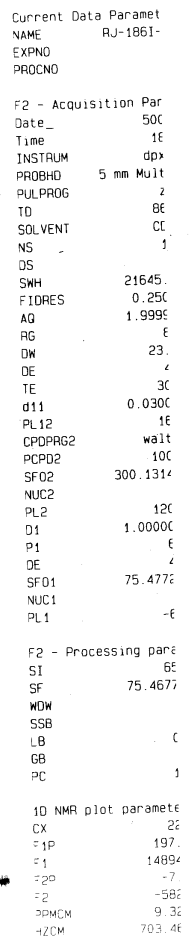

言

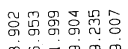

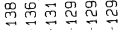

농ํㅇ

$\mathrm{Ph} \mathrm{NO}_{2}$

entry-11

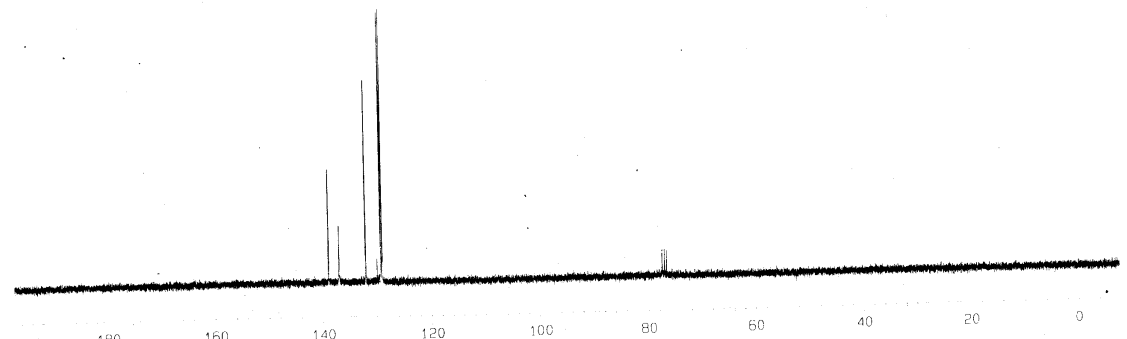

言

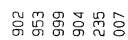

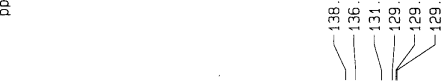

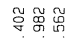

$\mathrm{Ph}_{\mathrm{CN}}$

entry-12
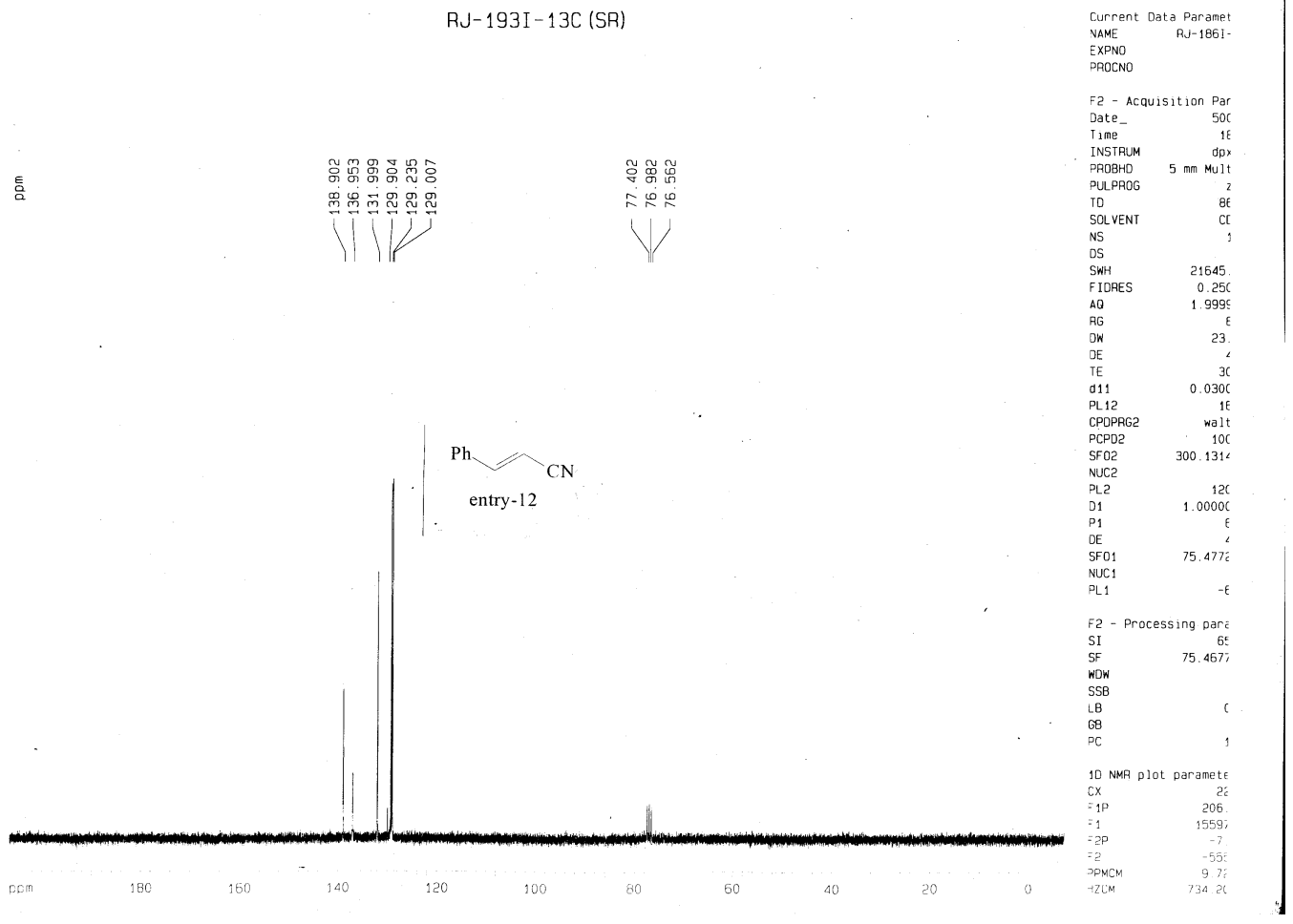
RJ-103E-13C (SR)
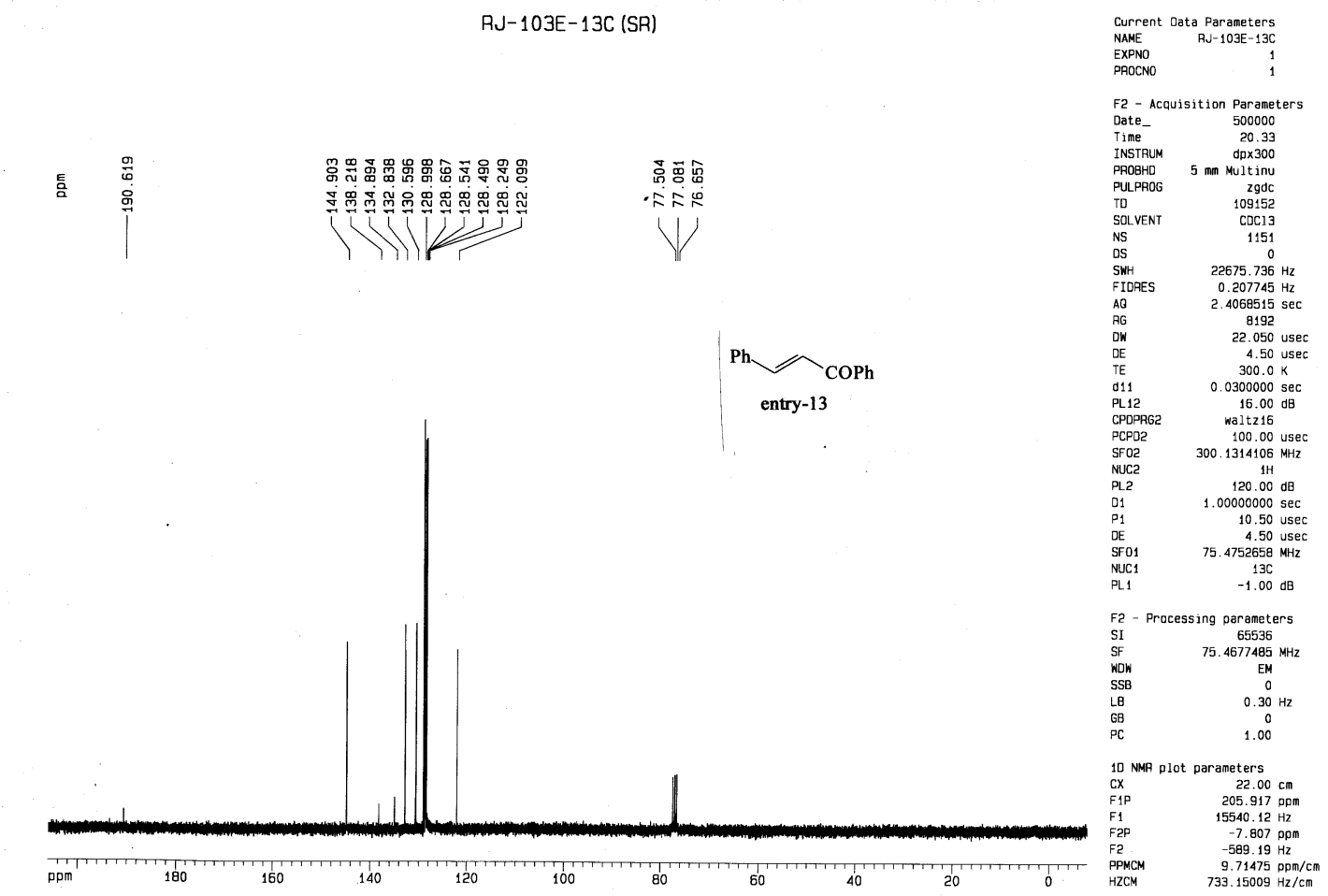

RJ-142I-13C (SR)
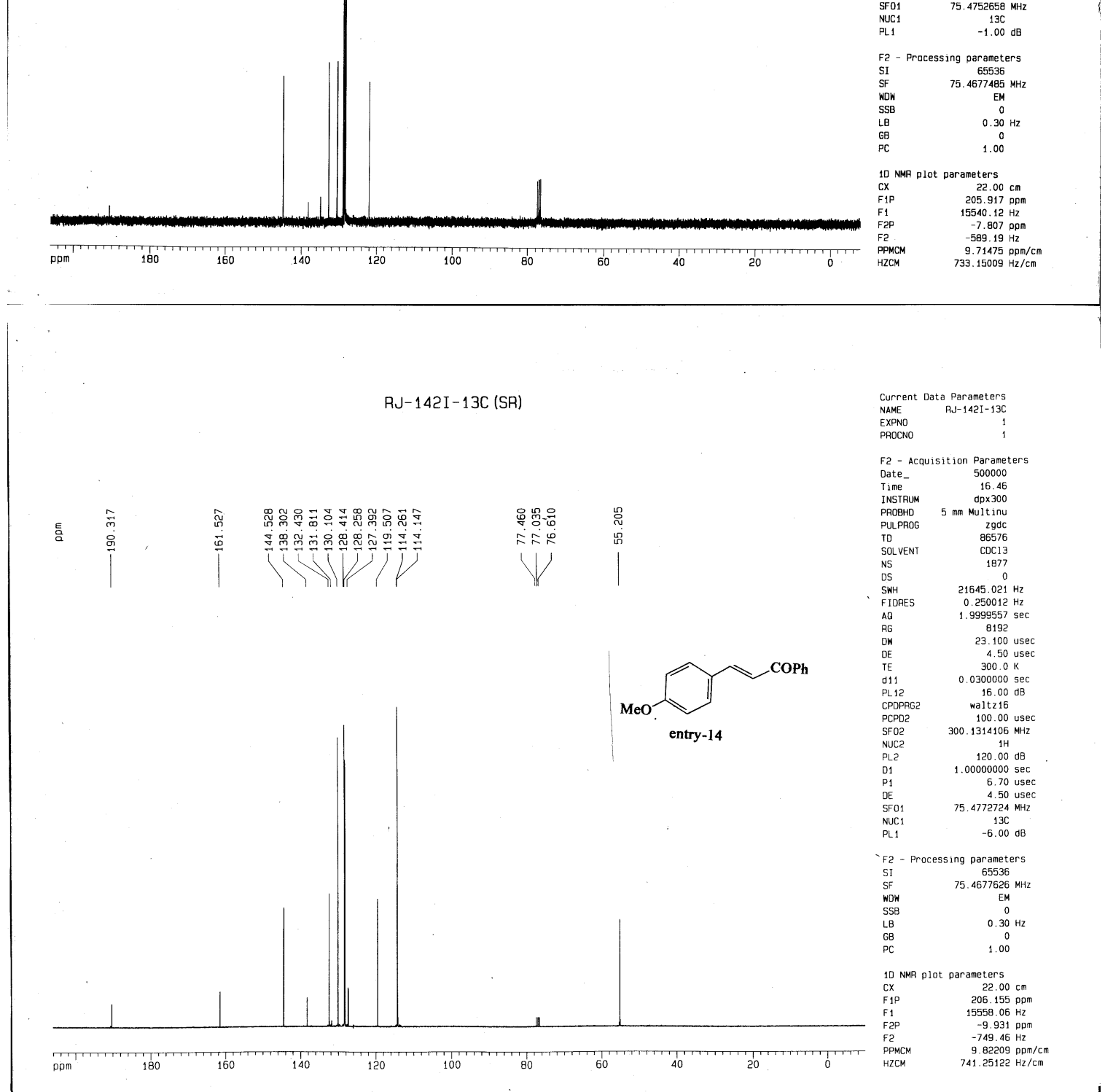
$R J-49 I-13 C$ (SR)

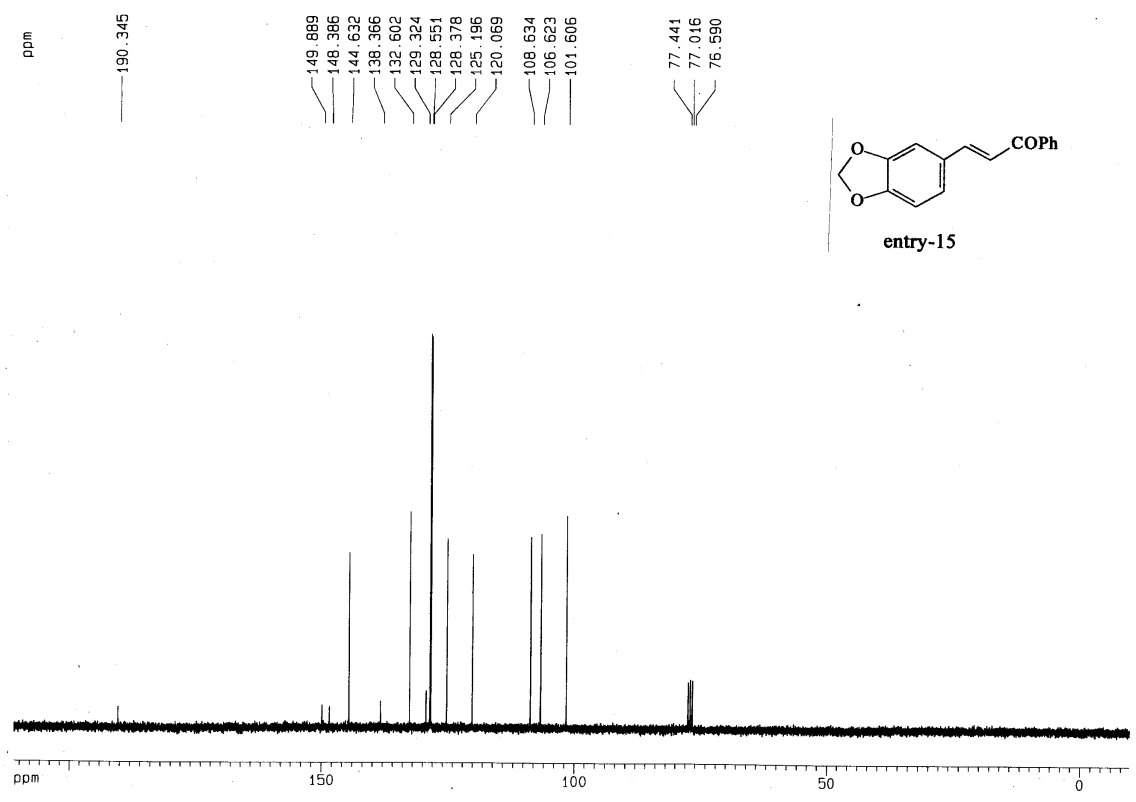

RJ-56I-13C (SR)

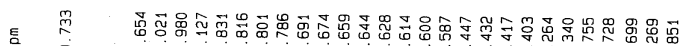
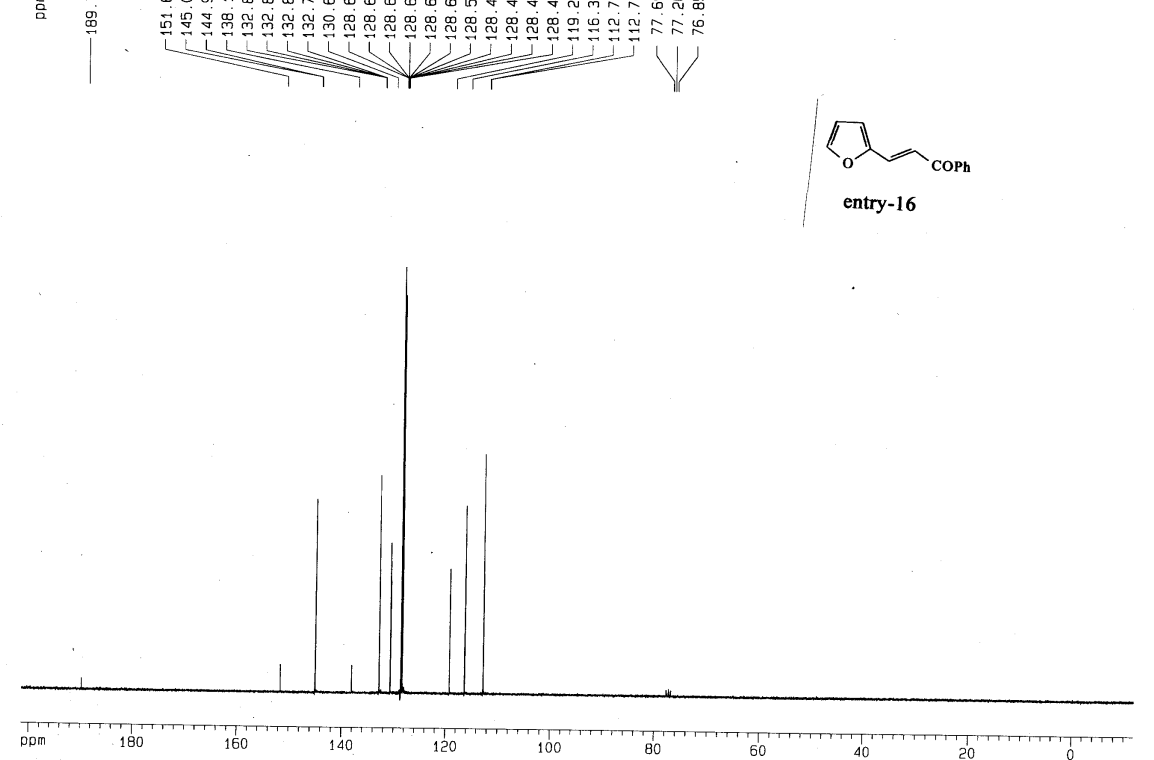
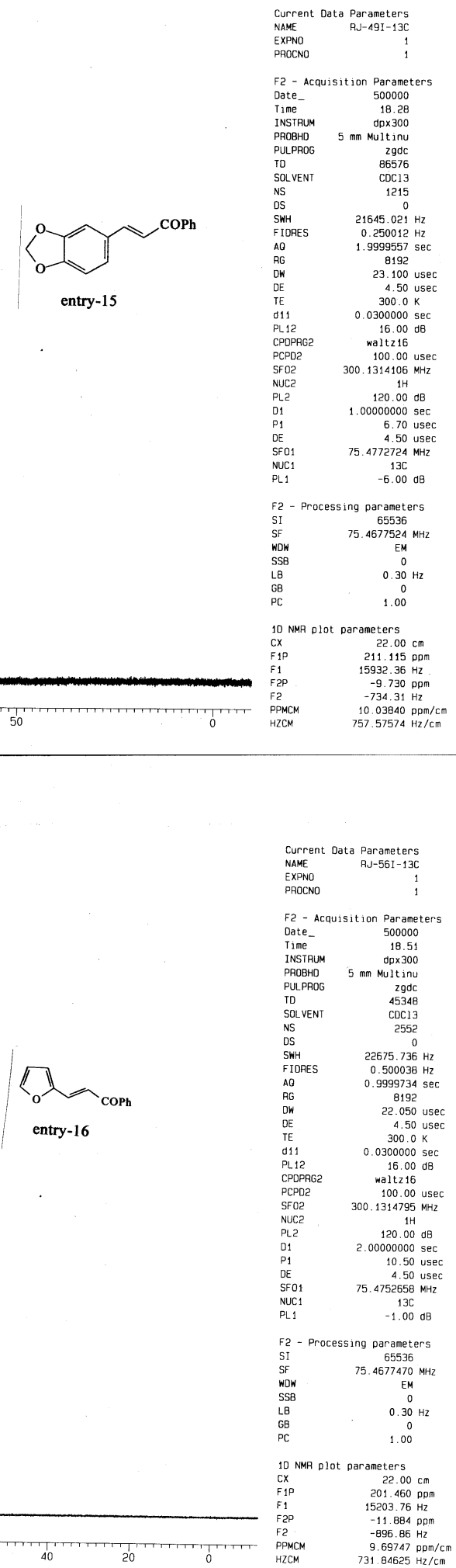
RJ-45I-13C (SR)

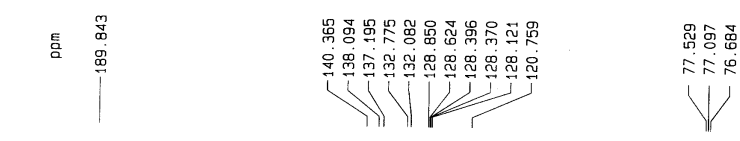

RJ-141I-13C (SR)
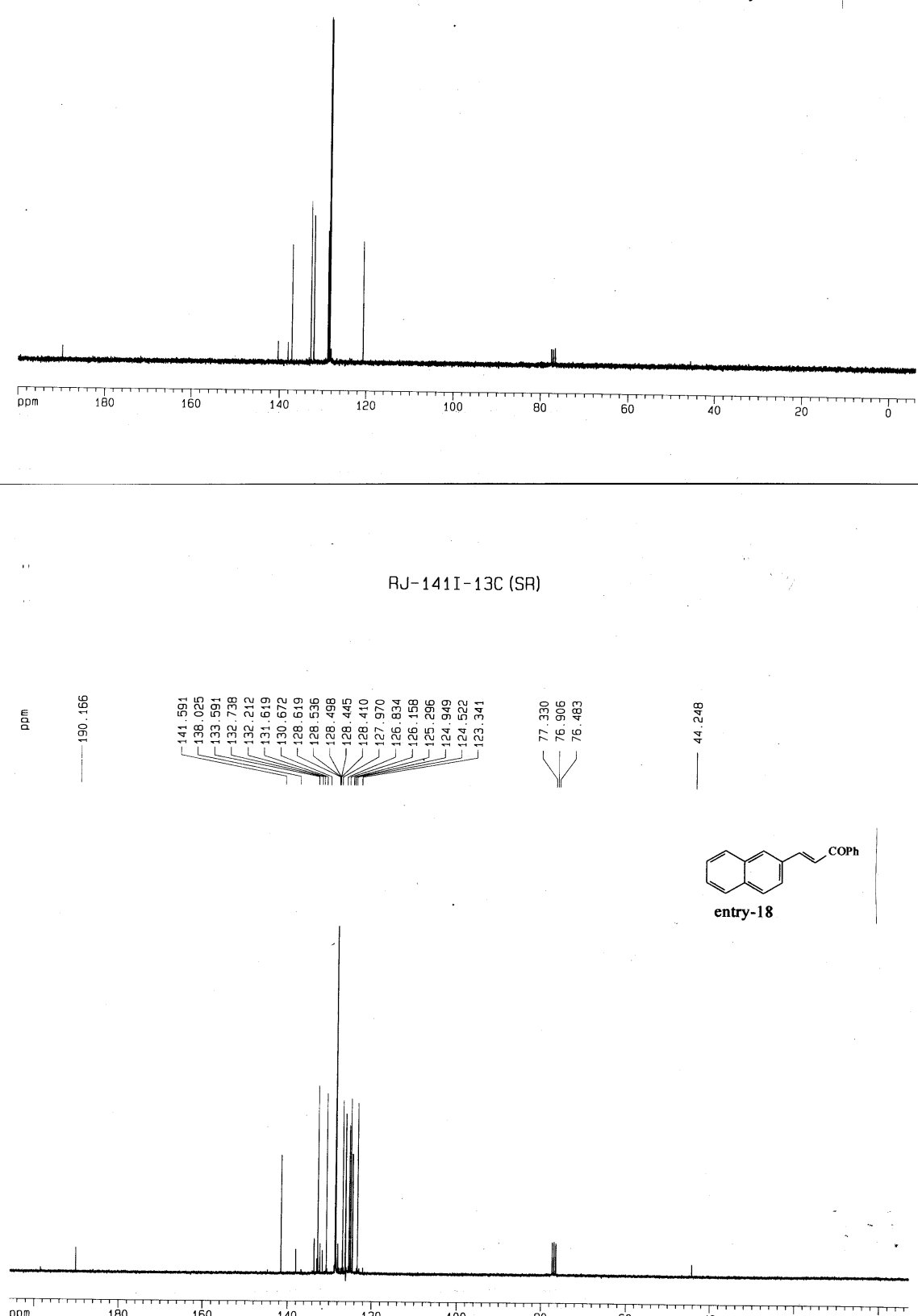

ppm

60
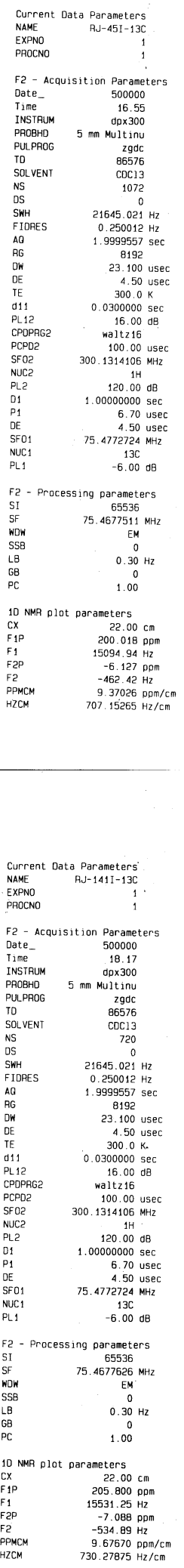

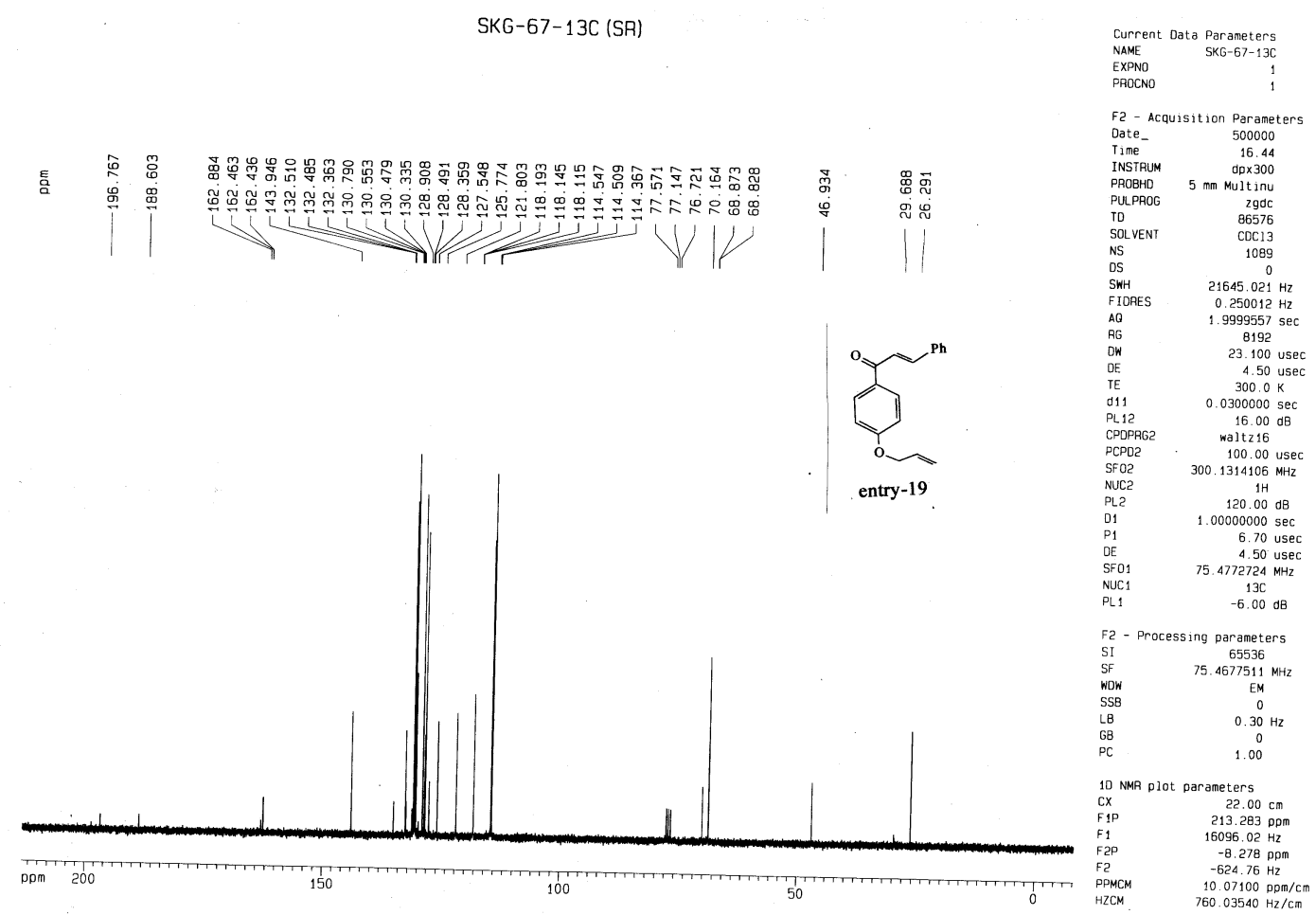

$R J-107 F-13 C$ (SR)

言
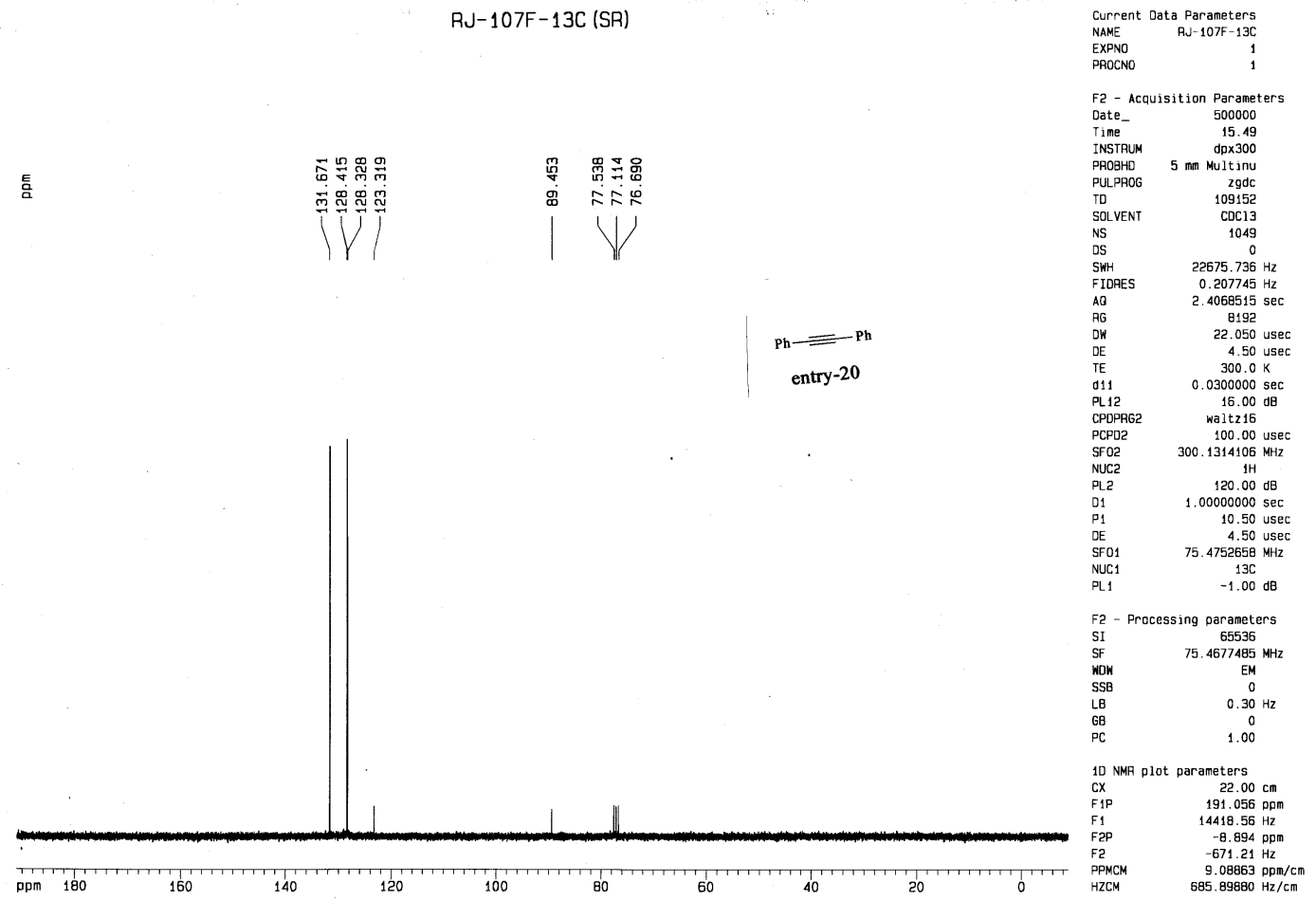


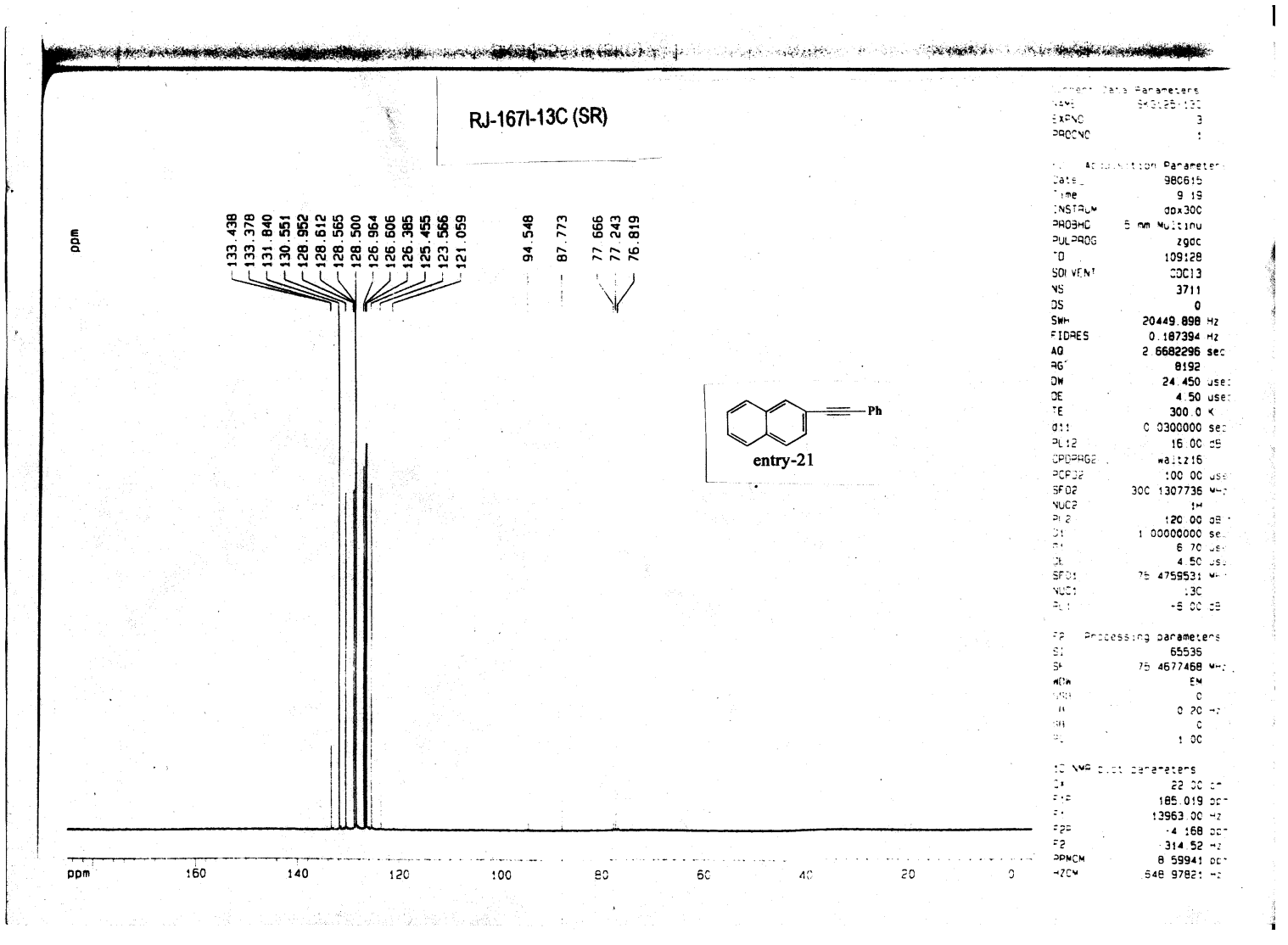

RJ $-42 I-1-13 C$ (SR)
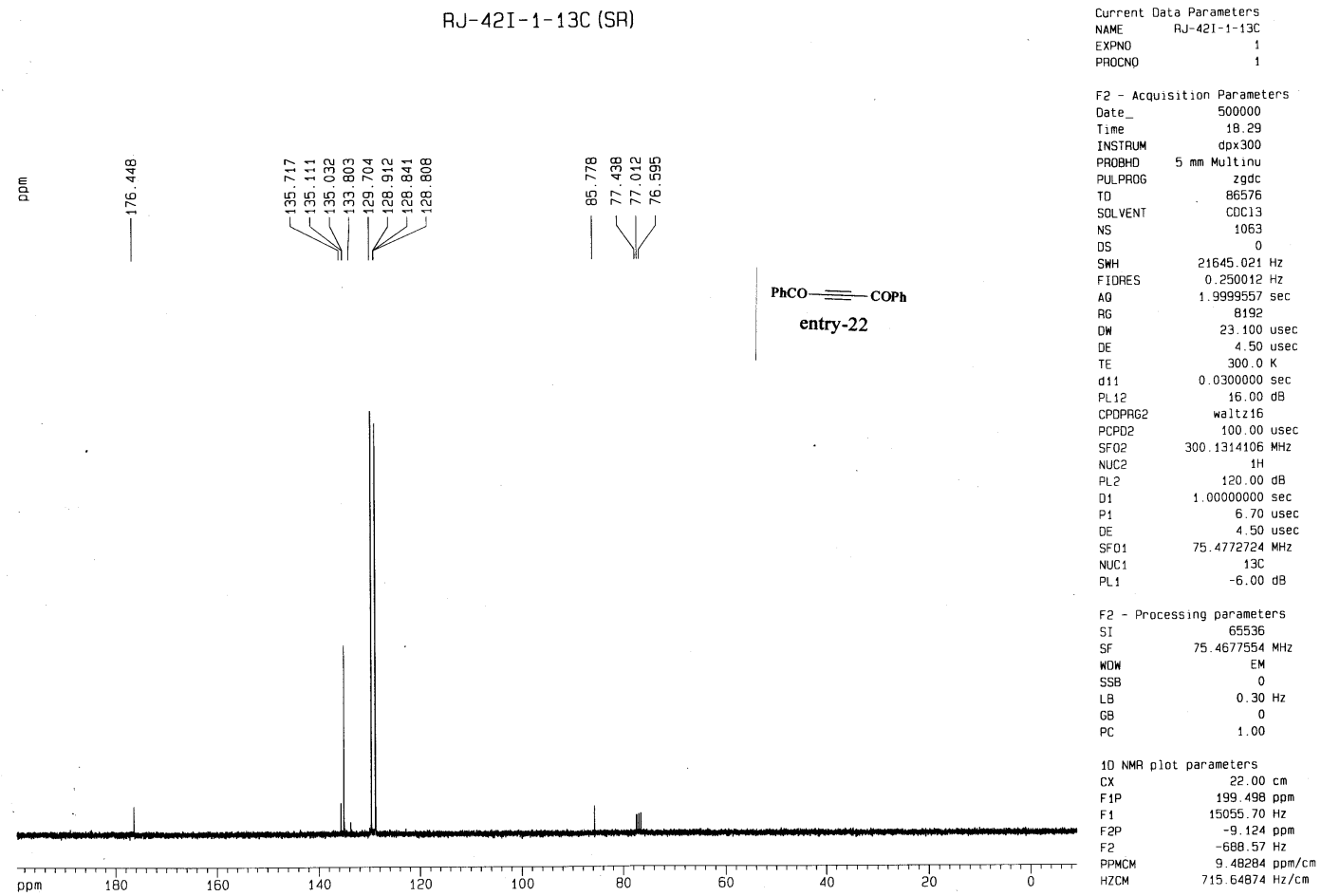
R3-50I-13C (akm)

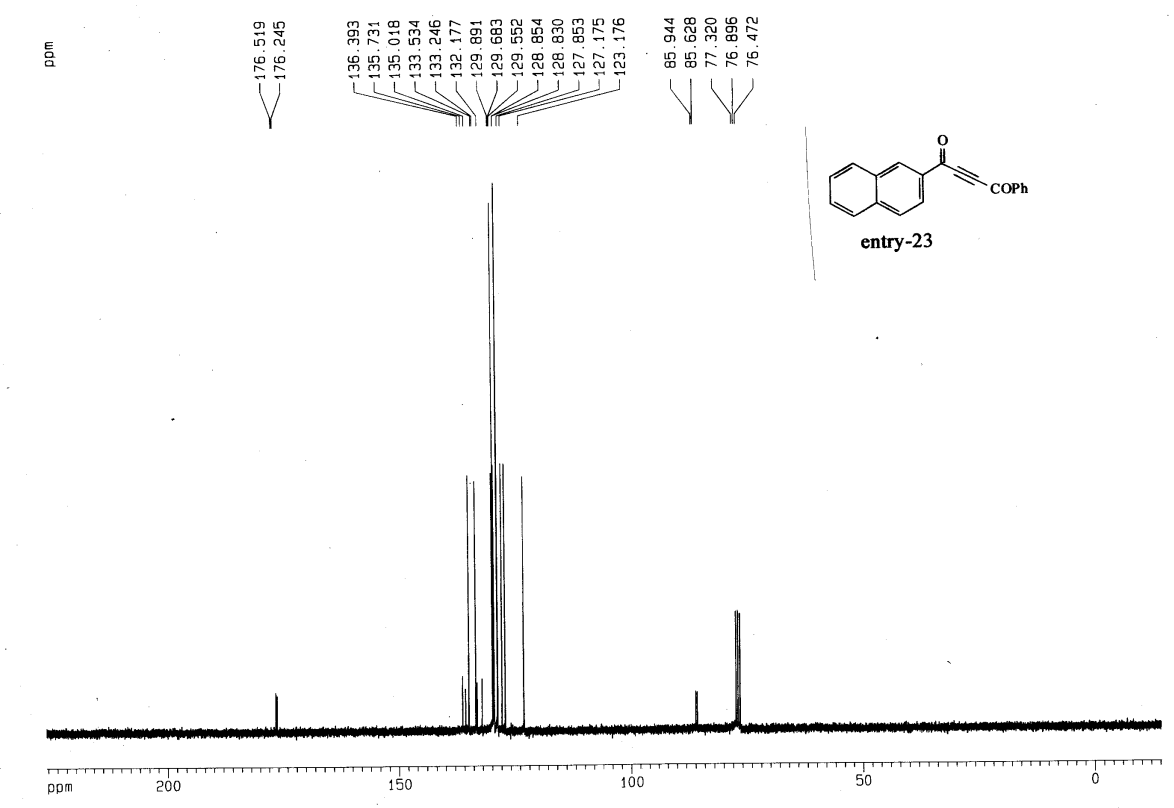

RJ-60I-13C (SR)
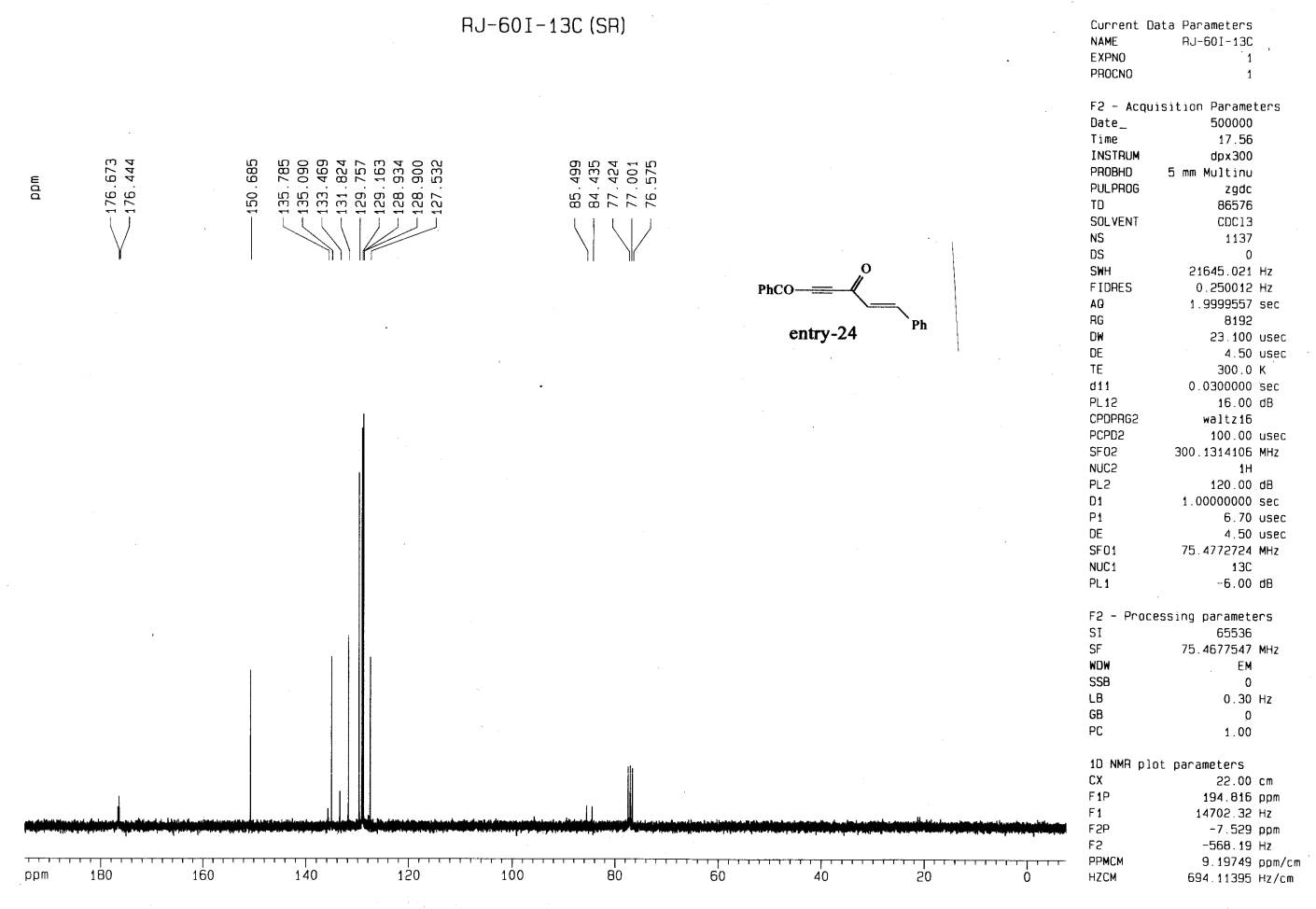

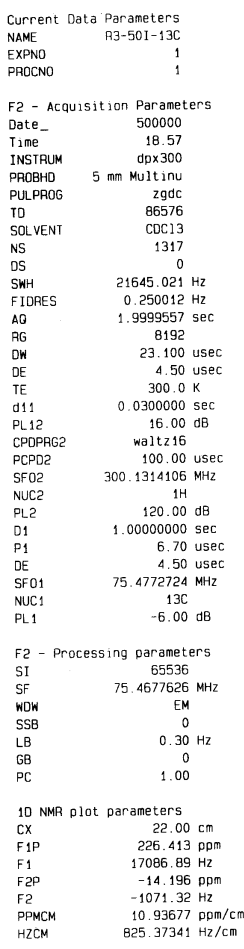


RJ37I-13C (SKD)
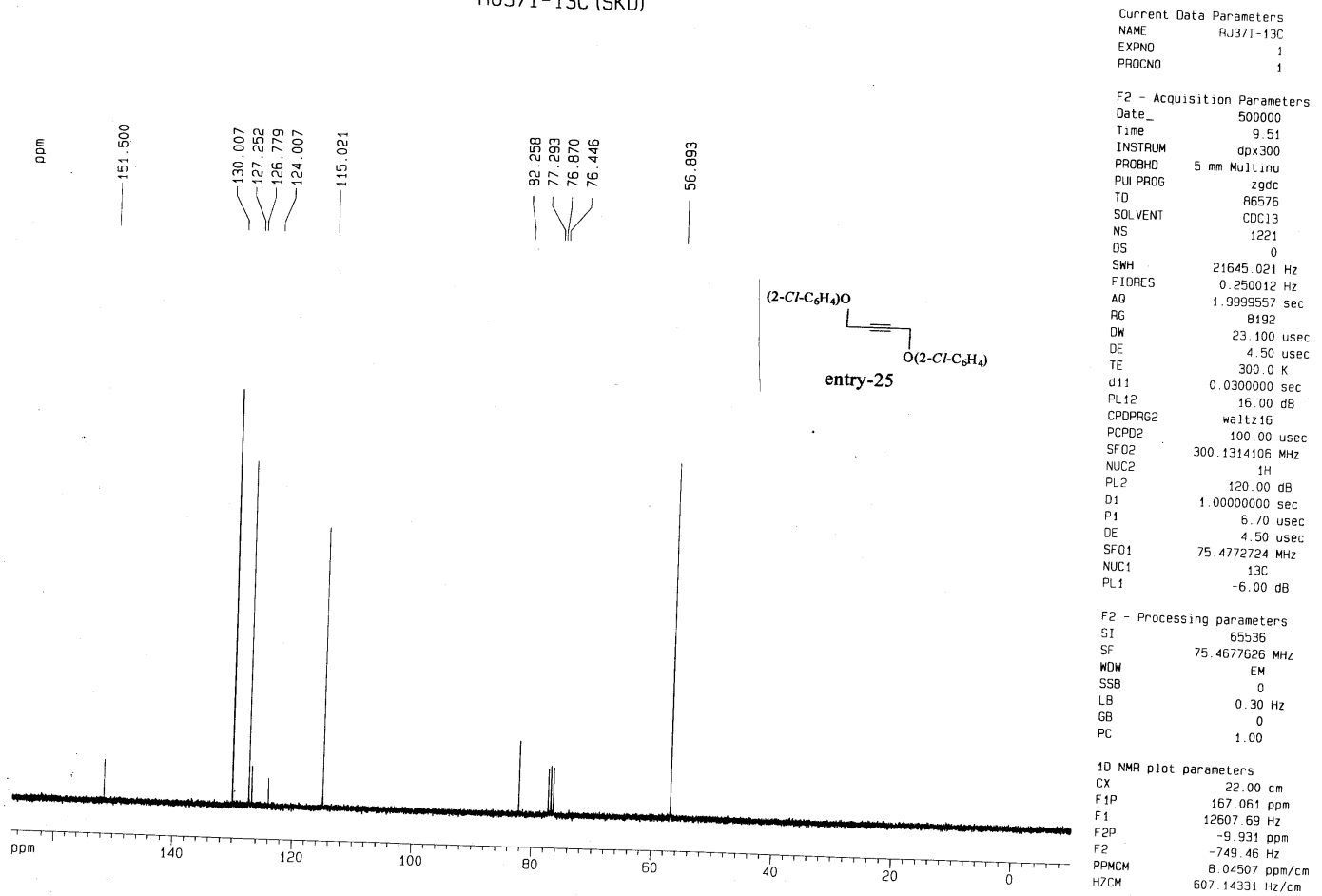
